\title{
El trastorno por estrés postraumático: una consecuencia de los asaltos
}

\author{
Carmen G loria Carbonell Ma , César Carvajal A. \\ Post traumatic stress disorder among \\ assault victims
}

Background: Urban violence is increasing in Metropolitan Santiago and assault victims may suffer from post traumatic stress disorders. Aim: To study the clinical and evolutive profile of post traumatic stress disorders among assault victims. Material and methods: Retrospective review of medical records of assault victims with post traumatic stress disorder, that were attended at the Hospital del Trabajador between 1987 and 2000. The features of these patients were compared with other patients with post traumatic stress disorders not caused by assaults. Results: The records of 140 subjects ( 88 female) aged $34.9 \pm 10.9$ years old, were reviewed. Seventy one percent of subjects worked as clerks and $59 \%$ were attended during the first month after the assault. Treatment with psychotherapy and psychotropic drugs lasted a median of 60 days and $62 \%$ was discharged before completing three months of treatment. These subjects used more antidepressants and had more physical lesions than subjects with a post traumatic stress disorder not caused by assaults. Conclusions: Timely diagnosis and treatment of post traumatic stress disorders caused by assaults, results in a favorable outcome in two thirds of patients (Rev Méd Chile 2004; 132: 839-44).

(Key Words: Crime; Stress disorders, post-traumatic; Violence)

Recibido el 16 de octubre, 2003. Aceptado en versión corregida el 27 de abril, 2004. Trabajo patrocinado por la Fundación Científica y Tecnológica ACHS. Proyecto 2299. Servicio de Salud Mental, Hospital del Trabajador, Santiago.

apsicóloga.

$\mathrm{A}$ ctualmente, los sucesos catalogados como traumáticos están integrados a nuestra vida, debido, en parte, al poderoso efecto de los medios de comunicación, lo que nos obliga a tomar conciencia de ellos. Se reconocen por su naturaleza disruptiva y sus consecuencias, son peligrosos e inesperados, y varían en intensidad y duración. Pueden afectar a individuos aislados o a comunidades, y pueden ser causados por la naturaleza (desastres naturales) o provocados por el hombre ${ }^{1}$.

Correspondencia a: P Carmen Gloria Carbonell. Vicuña Mackenna 200 tercer piso, Providencia. Santiago de Chile. Fono: 6853722. E-mail: carmengcarbonell@yahoo.es ghtrae@gw,achs.cl
Los actos de violencia constituyen un tema de gran importancia para la población general y las autoridades, tanto por sus consecuencias en el ámbito de la salud, como por las pérdidas que implican a nivel económico. Los asaltos a las personas en la vía pública generan una percepción de inminente peligro y desprotección. De acuerdo a las estadísticas, 83\% de la población estadounidense será víctima de algún delito violento a lo largo de su vida y el 99\% experimentará un robo ${ }^{2}$.

Cuando un individuo sufre un asalto, presenta una serie de reacciones a nivel psicológico y fisiológico debido a la vivencia de amenaza a la integridad personal; se experimenta el riesgo de 
perder la vida o sufrir lesiones físicas. Las creencias de control y omnipotencia acerca de sí mismo y del mundo son cuestionadas en forma dramática, generando respuestas que buscan enfrentar la sensación de vulnerabilidad interna. Creer que las personas obtienen lo que merecen o sentir que el mundo es un lugar seguro, son creencias que se frustran (hay pérdida de fe y sentimiento de desesperanza) ${ }^{3}$.

A esta amenaza a la integridad individual se suma la intencionalidad del agresor, lo que causa en la víctima sentimientos de rabia e impotencia por la percepción de injusticia y desamparo, llevando muchas veces a la idea de vengarse por lo sucedido.

Las respuestas inmediatas ante un suceso traumático serán diferentes en cada individuo y variarán según la naturaleza y consecuencias de la agresión. En la primera etapa, o de shock, que puede durar desde algunos minutos a días, el sistema defensivo del sujeto se activa ante el peligro, lo que puede facilitar o entorpecer el enfrentamiento a la situación y la posterior reacción emocional. La respuesta al trauma estará dada por la percepción de la amenaza y el significado que adquiera el hecho traumático; dicha percepción dependerá del acontecimiento en sí mismo, como de las caractenísticas de la persona ${ }^{4-6}$.

La respuesta individual dependerá también de la existencia de lesiones físicas y de la necesidad de atención médica a causa de las lesiones. Las hospitalizaciones, tratamientos invasores y de rehabilitación que se requieran, podrían determinar variaciones en la respuesta emocional del sujeto. La relación entre la severidad de la lesión y los síntomas postraumáticos es bastante variable; se podría esperar que a mayor gravedad de la lesión fuera mayor la percepción subjetiva de amenaza a la vida, lo cual aumentaría la probabilidad de desarrollar alteraciones psicológicas ${ }^{7}$.

Una vez superada la etapa de shock puede aparecer una respuesta emocional que se caracteriza por horror e indefensión. Breslau et al encontraron que 22,6\% de los sujetos expuestos a un asalto desarrollaron un Trastorno por Estrés Postraumático (TEPT) ${ }^{8}$.

Los síntomas primordiales del TEPT se caracterizan por una tríada con síntomas invasores, de evitación y de hiperalerta ${ }^{9}$. Pueden presentarse recuerdos recurrentes del hecho, pesadillas, evitación de conversaciones, personas o lugares que evoquen el trauma, alteraciones del sueño, irritabilidad e intensa angustia.
No todas las personas expuestas a un acontecimiento traumático desarrollan un TEPT. Diversos estudios plantean que sólo la mitad de los sujetos presentarán síntomas característicos del TEPT ${ }^{10,11}$.

El TEPT reflejaría la mantención de la vivencia disruptiva y del impacto emocional que ha causado el asalto en la persona. Después del asalto el paciente se enfrenta a un mundo peligroso y no es capaz de modificar su conducta ante las contingencias externas ${ }^{4}$. No sólo está temeroso, confuso y con rabia, sino que además, se encuentra en una vivencia constante de vulnerabilidad al revivir el acontecimiento traumático.

Una parte importante de los pacientes atendidos en el Servicio de Salud Mental del Hospital del Trabajador de Santiago (HTS) por TEPT han sido víctimas de un asalto en el trayecto desde su trabajo al hogar, o viceversa, siendo por esta razón acogidos por la Ley de Accidentes del Trabajo $16.744^{12}$. Cuando ingresan al Servicio de Salud Mental son evaluados por un médico psiquiatra, quien precisa el diagnóstico, indica el tratamiento farmacológico y, en caso de requerir psicoterapia, los deriva a una atención psicológica.

El tratamiento psiquiátrico se fundamenta en un modelo médico con orientación psicoterapéutica de tipo cognitivo-conductual, cuyo objetivo final es el control de los síntomas y la pronta reinserción laboral y sociofamiliar.

Debido a las repercusiones personales, laborales y familiares que tienen estos hechos traumáticos, se decidió estudiar el perfil clínico y evolutivo de los pacientes con la intención de detectar factores como: sexo, lesiones físicas, características de personalidad y del tratamiento que pueden influir en la evolución, para así poder ofrecer la terapia más adecuada.

\section{MATERIAL Y MÉTODO}

Se revisaron las fichas clínicas de pacientes ingresados al Servicio de Salud Mental del HTS entre los años 1987 y 2000, con el diagnóstico de TEPT de acuerdo a los criterios del DSM-IV ${ }^{9}$. De un total de 450 fichas se seleccionaron aquellas en que el acontecimiento traumático fue un asalto en la vía pública $(31,0 \%)$.

Se elaboró una ficha de registro en que se consignaron diversas variables de importancia demográfica y clínica. 
Estadística. El análisis de las variables estudiadas se realizó mediante el programa computacional Epi-Info versión 6.04. Se compararon las variables estudiadas con la muestra total de pacientes con TEPT para determinar el perfil específico de los pacientes que habían tenido como estresor un asalto.

\section{RESULTADOS}

La muestra de 140 pacientes con TEPT víctimas de un asalto estuvo constituida por 88 mujeres $(62,9 \%)$, con edad de $34,9 \pm 10,9$ años $(\bar{x} \pm \mathrm{DE}) \mathrm{y}$

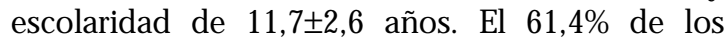
pacientes estaba casado al momento del asalto y trabajaban predominantemente en actividades de servicio $(71,4 \%)$ (Tabla 1$)$.

El 59,0\% de los pacientes recibió la atención inicial en el Servicio de Salud Mental dentro del primer mes desde la ocurrencia del hecho traumático $(20,7 \%$ de la muestra fue atendida en la primera semana después del asalto) (Figura 1).

La primera atención fue de tipo ambulatoria en 135 pacientes $(96,4 \%)$. Sólo $3,6 \%$ de ellos se encontraba hospitalizado al momento de la primera consulta psiquiátrica producto de las lesiones físicas. El 64,3\% de los pacientes sufrió algún tipo de lesión física, a raíz de la agresión provocada durante el asalto; las más frecuentes fueron las contusiones ( $40 \%$ del total de lesiones), seguidas por heridas por arma blanca $(25,6 \%)$ y fracturas (20\%), por lo que estos pacientes requirieron tratamiento médico o quirúrgico paralelo a la intervención de salud mental.
Los síntomas de TEPT más frecuentemente referidos fueron -además de los síntomas invasores y de evitación, ambos presentes en $100 \%$ de los pacientes- ansiedad (97,9\%), insomnio de conciliación (88,6\%), pesadillas con el acontecimiento traumático $(71,4 \%)$, síntomas de hiperalerta $(78,6 \%)$ y exagerada activación del sistema nervioso autónomo ante cualquier estímulo relacionado con el asalto (73,6\%) (Tabla 2).

La evaluación psicopatológica, realizada por los psiquiatras del HTS a través de la entrevista clínica habitual, permitió además explorar la personalidad premórbida. Siguiendo los criterios del borrador de

Tabla 1. C aracterísticas demográficas de $\mathbf{1 4 0}$ pacientes con T EPT víctimas de un asalto

\begin{tabular}{|lcc|}
\hline & $\mathrm{n}$ & $\%$ \\
\hline Sexo & & \\
$\quad$ Mujeres & 88 & 62,9 \\
Hombres & 52 & 37,1 \\
Edad (años) & & \\
$\quad(\overline{\mathrm{x}} \pm \mathrm{DE})$ & $34,9 \pm 10,9$ & \\
Escolaridad (años) & & \\
$\quad(\overline{\mathrm{x}} \pm$ DE) & $11,7 \pm 2,6$ & \\
Estado Civil & & \\
Soltero & 50 & 35,7 \\
Casado & 86 & 61,4 \\
Separado-Viudo & 4 & 2,9 \\
Ocupación & & \\
Servicios & 100 & 71,4 \\
Industria & 27 & 19,3 \\
Otros & 13 & 9,3 \\
\hline
\end{tabular}

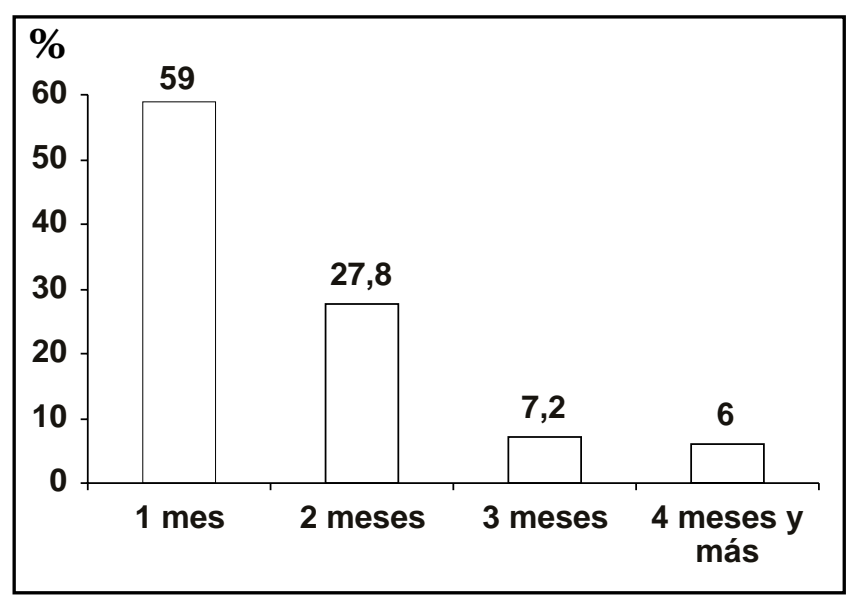

Figura 1. Tiempo de derivación a salud mental desde el momento del asalto. 
la CIE-10 que circunscribe la sacentuación de rasgos de personalidad», se encontró que esta categonía estaba presente en 19,3\% de los pacientes. El 7,1\% de la muestra cumplió con los criterios diagnósticos de la CIE-10 para trastomo de personalidad.

El 98,6\% de los casos recibió algún tipo de fármaco: benzodiacepinas en 97,1\%, antidepresivos en $47,1 \%$ y neurolépticos en $17,9 \%$ de los pacientes. Las mujeres recibieron más antidepresivos $(59,1 \%$ vs $28,8 \%, p<0,05)$ y más neurolépticos $(23,9 \%$ vs $7,7 \%, p<0,05)$ que los hombres.

La psicoterapia se indicó al $80,0 \%$ de la muestra $(n=112)$. Entre las técnicas específicas utilizadas se realizó psicorrelajación en 66 pacientes $(47,5 \%)$ y desensibilización en imaginería en 49 pacientes $(35,3 \%)$.

El tratamiento en salud mental tuvo una duración de 70 días (mediana); a los tres meses, el $64,4 \%$ de la muestra había sido dado de alta. Se requirió un promedio de 5,2 sesiones con psiquiatra y 5,1 sesiones con psicóloga. Al alta, el 65,2\% de los pacientes estaba asintomático y el resto presentaba síntomas leves, que no constituían una limitante para trabajar (Figura 2).

Las mujeres requirieron mayor tiempo de tratamiento que los hombres; a los tres meses, $61,6 \%$ de las mujeres habían sido dadas de alta de los controles en comparación con $71,2 \%$ de los hombres $(\mathrm{p}<0,05)$ (Figura 3). Además, se indicó más antidepresivos a las mujeres que a los hombres (59,1\% vs $28,8 \%$, p <0,05); en cambio los hombres utilizaron más neurolépticos que las mujeres $(7,7 \%$ vs $3,9 \%, p<0,05)$.

La segunda parte del estudio comparó las características demográficas, clínicas y de la evolu- ción de los pacientes con TEPT víctimas de asalto $(n=140)$, con los pacientes con TEPT de otro origen (accidente automovilístico, abuso sexual y violación, y accidentes ocurridos en el lugar de trabajo) ( $\mathrm{n}=310)$ para obtener un perfil específico de los pacientes que fueron asaltados.

En el grupo que sufrió un asalto hubo un mayor porcentaje de mujeres (62,9\% vs 50,3\%; $p$ $<0,05)$ y predominó el trabajo en actividades de servicio $(71,4 \%$ vs $55,2 \%$; $p<0,05)$ respecto al grupo con TEPT a raíz de otros estresores. Las lesiones físicas y el empleo de fármacos antidepresivos fueron más frecuentes (diferencias esta-

Tabla 2. Características clínicas de 140 pacientes con T EPT víctimas de un asalto

\begin{tabular}{|lrc|}
\hline & $\mathrm{n}$ & $\%$ \\
\hline Lesiones físicas & 90 & 64,3 \\
Atención inicial & & \\
$\quad$ Ambulatoria & 135 & 96,4 \\
$\quad$ Hospitalización & 5 & 3,6 \\
Síntomas & & \\
$\quad$ Ansiedad & 137 & 97,9 \\
$\quad$ Alteraciones del sueño & 124 & 88,6 \\
$\quad$ Síntomas de hiperalerta & 110 & 78,6 \\
$\quad$ Síntomas autonómicos & 103 & 73,6 \\
$\quad$ Pesadillas & 100 & 71,4 \\
Personalidad & & \\
$\quad$ Normal & 103 & 73,6 \\
$\quad$ Anormal* & 37 & 26,4 \\
\hline
\end{tabular}

*Incluye trastornos de personalidad y acentuación de rasgos de la personalidad.

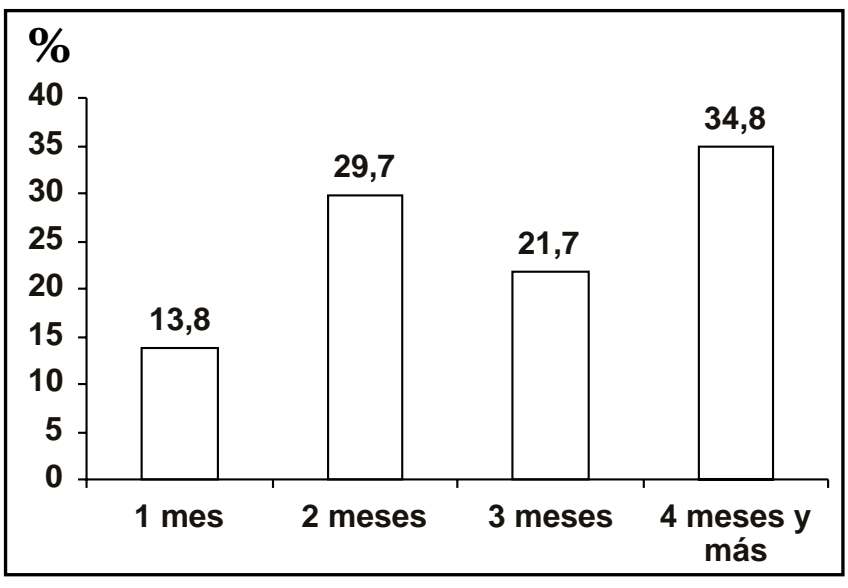

Figura 2. Tiempo de tratamiento en salud mental de 140 pacientes con TEPT víctimas de un asalto. 


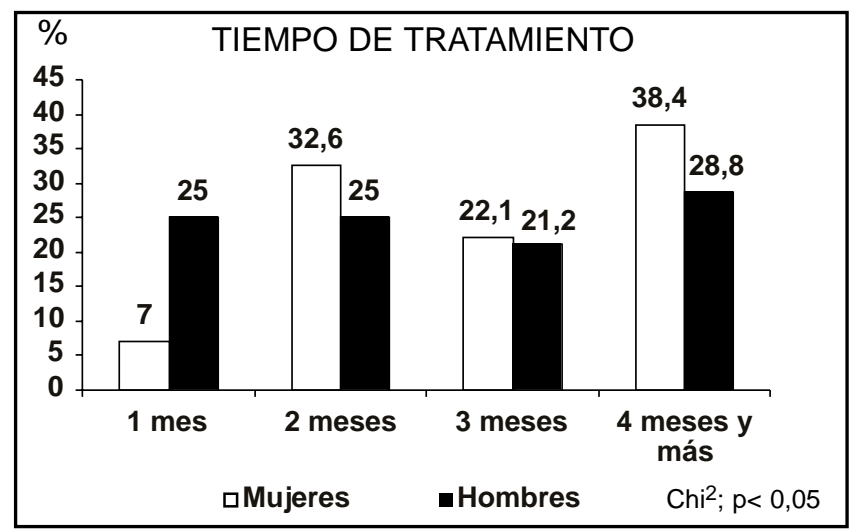

Figura 3. Diferencias significativas por sexo de 140 pacientes con TEPT víctimas de un asalto.

dísticamente significativas) en los pacientes con TEPT de otro origen que entre los pacientes con TEPT producto de un asalto. Las otras variables estudiadas no tuvieron diferencias estadísticamente significativas (Tabla 3).

\section{DisCUSIÓN}

La muestra estudiada corresponde a una población de trabajadores activos acogida a la Ley de Accidentes del Trabajo, en que el asalto constituye un tipo de accidente de trayecto, al ocurrir en el traslado del trabajador desde su casa a la empresa o fábrica, o viceversa.

Debido a las características de la ley y al tipo de atención entregada en el Hospital del Trabajador (que la mayoría de las veces es inmediata 0 muy cercana a la ocurrencia del hecho denunciado), es posible detectar precozmente los síntomas de TEPT y derivar oportunamente a los pacientes para su tratamiento.

Los resultados demuestran que las víctimas de asalto con TEPT son de preferencia jóvenes, con buen nivel de escolaridad, observándose un mayor predominio de mujeres. Las mujeres tienen mayor riesgo de exposición a un asalto que a otro tipo de hecho traumático, y además presentan una mayor probabilidad de desarrollar un TEPT después de este tipo de suceso (referencia $13 \mathrm{y}$ documento: «iagnóstico de la Delincuencia: Antecedentes sobre la delincuencia en Chile». Fundación Paz Ciudadana. Mayo, 2001). Breslau et al, encontraron que entre los estresores que provocan TEPT en las mujeres los asaltos y las violaciones o abusos sexuales (27\%) fueron más
Tabla 3. Variables con diferencias significativas entre pacientes con TEPT víctimas de un asalto y por otros estresores

\begin{tabular}{|c|c|c|c|c|}
\hline & \multicolumn{2}{|c|}{ Asalto } & \multicolumn{2}{|c|}{ Otros } \\
\hline & $\begin{array}{c}\mathrm{n} \\
(140)\end{array}$ & $\%$ & $\begin{array}{c}\mathrm{n} \\
(310)\end{array}$ & $\%$ \\
\hline \multicolumn{5}{|l|}{ Sexo* } \\
\hline Mujeres & 88 & 62,9 & 156 & 50,3 \\
\hline Hombres & 52 & 37,1 & 154 & 49,7 \\
\hline \multicolumn{5}{|l|}{ Ocupación** } \\
\hline Servicios & 100 & 71,4 & 171 & 55,2 \\
\hline Industria & 27 & 19,3 & 86 & 27,7 \\
\hline Otros & 13 & 9,3 & 53 & 17,1 \\
\hline Lesiones físicas** & 89 & 64,3 & 256 & 82,6 \\
\hline Antidepresivos* & 67 & 47,9 & 181 & 58,4 \\
\hline
\end{tabular}

Chi2; *p $<0,05, * *_{p}<0,001$.

frecuentes en comparación con los hombres $(8 \%)^{14}$. Por otra parte el TEPT, al igual que otros trastornos de ansiedad, tiene mayor incidencia en mujeres que en hombres, a pesar de que la probabilidad de experimentar algún hecho traumático en la vida es mayor para los hombres ${ }^{15,16}$.

Entre las características demográficas se pudo observar una alta proporción de pacientes que trabajaban en actividades de servicio, siendo mayor esta proporción que en los pacientes con TEPT de otro origen. Una parte importante de las actividades de servicio incluye varios desplazamientos diarios y gran exposición a la vía pública; esto se traduce en un mayor riesgo de sufrir asaltos en ese lugar, en comparación con los sujetos que pasan toda su jornada laboral en el 
mismo lugar de trabajo y que sólo realizan un traslado diario desde y hacia sus hogares. Esto coincide además con el aumento creciente de asaltos en la ciudad de Santiago en los últimos años (Documento: Evolución de la Delincuencia en Chile. Fundación Paz Ciudadana. Abril 2003), y con mayor porcentaje de ingresos por TEPT a salud mental a causa de un asalto: 17,2\% en 1988 y 41,3\% en el año 2000.

Las lesiones físicas no constituyeron un elemento distintivo en los pacientes asaltados, por lo que se puede considerar que la percepción de amenaza podría estar más bien vinculada al significado subjetivo que la persona le otorga al hecho traumático y a las características particulares del asalto, relacionadas con la intencionalidad de la agresión. Estos elementos exacerbarían la vivencia de daño y desamparo que un asalto provoca en el individuo.

\section{REFERENCIAS}

1. Ursano R, Fulerton C, McCaughey B. Trauma and disaster. En: Ursano RJ, McCaughay BG, Fullerton CS eds. Individual and community responses to trauma and disaster. Cambridge University Press. Cambridge. 1994.

2. Cía A. Trastorno por Estrés Postraumático: Diagnóstico y Tratamiento Integrado 1a Edición. Buenos Aires. Imaginador, 2001.

3. Ursano R, McCaughey B, Fulierton C. The structure of human chaos. En: Ursano RJ, McCaughay BG, Fullerton CS eds. Individual and community responses to trauma and disaster. Cambridge University Press. Cambridge. 1994.

4. Harvey P, Yehuda R. Strategies to study risk for the development of PTSD En Yehuda R (ed), Risk factors for posttraumatic stress disorder. American Psychiatric Press Inc. 1st Edition, Washington, DC. 1999.

5. CaRBonell CG. Trastorno por estrés postraumático: clínica y psicoterapia. Rev Chil Neuro-Psiquiat 2002; 40 (supl 2): 69-75.

6. Carvajal C. Trastorno por estrés postraumático: aspectos clínicos. Rev Chil Neuro-Psiquiat 2002; 40 (supl 2): 20-34.

7. Michaels AJ, Michaels CE, Smith JS, Moon CH, Peterson C, Long WB. Posttraumatic Stress Disorder in injured adults: etiology by path analysis. J Trauma 1999; 47: 867-73.
Los resultados de este estudio confirmaron que un tratamiento combinado, que incluyó manejo farmacológico -principalmente uso de benzodiacepinas- y psicoterapia cognitivo-conductual, permitió una evolución favorable en un período menor a tres meses. La mayoría de los pacientes pudieron reinsertarse laboralmente y normalizar sus vidas. Las pacientes mujeres, a diferencia de los hombres, requirieron un mayor tiempo de tratamiento y más fármacos (antidepresivos y neurolépticos), lo que puede reflejar un mayor impacto emocional y más sentimientos de indefensión después de este tipo de trauma.

Aunque este estudio tiene limitaciones por su carácter retrospectivo, representa un importante avance para definir el perfil clínico de un grupo de pacientes que ha ido en aumento y necesita de ayuda especializada para recuperarse integralmente y dejar atrás el rótulo de síctima».

8. Breslau N, Davis G, Andreski P, Peterson E. Traumatic events and posttraumatic stress disorder in an urban population of young adults. Arch Gen Psychiatry 1991; 48: 216-22.

9. American Psychiatric Association. Diagnostic and Statical Manual of Mental Disorder. 4th ed. American Psychiatric Press. Washington, DC. 1994.

10. Wolf M, Mosnaim A. Post traumatic stress disorder: etiology, phenomenology and treatment. American Psychiatric Press Inc., Washington, DC. 1990.

11. McFariane A. The etiology of post-traumatic morbidity: predisposing, precipitating and perpetuating factors. Br J Psychiatry 1989; 154: 221-8.

12. Ley 16.477 de Accidentes del Trabajo y Enfermedades Profesionales.

13. Breslau N, Davis G, Andreski P, Peterson E, Schultz L Sex differences in Posttraumatic Stress Disorder. Arch Gen Psychiatry 1997; 54: 1044-8.

14. Bresiau N, Davis G, Peterson E, Schultz L Psychiatric sequelae of PTSD in women. Arch Gen Psychiatry 1997; 54: 81-7.

15. Kessler RC, Sonverge A, Bromet E, Nelson CB. Posttraumatic Stress Disorder in the National Comorbidity Survey. Arch Gen Psychiatry 1995; 52: 1048-50.

16. Brewin RC, Andrews B, Rose S, Kirk M. Acute stress disorder and posttraumatic stress disorder in victims of violent crime. Am J Psychiatry 1999; 156: 360-6. 